\title{
Parameters of heat treatment increased concrete strength at its watertightness
}

\author{
Nurmuhamat Asatov", Mirjalol Tillayev and Navruzbek Raxmonov \\ Djizak Polytechnic Institute, Construction of buildings and structures department, Djizak city, \\ Uzbekistan
}

\begin{abstract}
One of the effective methods of land irrigation in the Republic of Uzbekistan is carried out with the help of precast reinforced concrete trays of irrigation systems. In the process of operation, precast concrete trays are influenced by difficult operating conditions. As is known, to achieve high water resistance and concrete strength after heat treatment, it is necessary to take into account such factors as pre-exposure time, temperature rise rate, and isothermal warm-up time. However, the determining factor that has the greatest impact on the water resistance of concrete during heat treatment is the duration of the preliminary exposure and the rate of temperature rise, on which the values of internal stresses in concrete depend on heating. The paper gives the results of scientific research, which, being based on the studies of heat and moisture treatment of concrete in a laboratory steam chamber with automatic control of the regime. Scientific methods and techniques were used in the process of data processing and study findings: systematic approach, methods of system analysis and mathematical statistics. To determine the strength of concrete, cubic samples were made with dimensions of 100x100x100 mm, which were tested at the period of 1,3,7,28 days. After HMT compared with similar characteristics of normal hardening samples. The results of studies of the kinetics of increase in concrete strength depending on the heat treatment regimes are shown in the paper, that, despite the slight difference in the duration of the preliminary curing ( 1 and 2 hours), there is still a certain difference between them. The scientific novelty lies in the formulation, formalization and solving problems related to water resistance, frost resistance, and at the same time saves heat and power resources of trays.
\end{abstract}

\section{Introduction}

One of the effective methods of land irrigation in the Republic of Uzbekistan is carried out with the help of precast reinforced concrete trays of irrigation systems. In the process of operation, precast concrete trays are influenced by difficult operating conditions. The process of the flow of water under the influence of pressure, especially its bottom part, contributes to its significant moistening and even filtration of water through the walls. Therefore, for the manufacture of precast concrete trays used concrete increased water resistance.

\footnotetext{
${ }^{*}$ Corresponding author: asatov_nur@list.ru
} 
In the manufacture of precast concrete trays, thermal and moisture treatment of it is important. The steam for heat treatment of the trays is supplied to the thermoform of the roof-forming parabola, from its lower end side. In the process of raising the temperature, the form undergoes a significant deformation. It should be noted that the zone of maximum temperature coincides with the open surface formed by the loading opening, causing intensive migration of water into this zone and its evaporation. This, in turn, leads to the formation of directional capillaries, impairing the waterproofness, frost resistance and strength of concrete in the most critical part of the tray during the operation of the tray.

The only way to eliminate such a negative phenomenon is intensive steam supply in order to displace the vapor-air medium to completely fill the internal space of the mold with steam. However, such an intensive steam supply can lead to a sharp rise in temperature in the initial stage of heat treatment of the trays.

But, as practice shows, such intense heating occurs in the thermoform itself and, to a lesser extent, in the concrete mix. To equalize the temperature between the thermoform and the concrete mix, a certain time is required for which the concrete mix must acquire some initial strength and the purpose of the duration of the preliminary keeping must be established on this basis.

In the research of S.A.Mironov, B.A.Krylov, F.M.Ivanov and L.A.Malininoy was shown the possibility of obtaining concrete with high physicomechanical properties after heat treatment.

As is known, to achieve high water resistance and concrete strength after heat treatment, it is necessary to take into account such factors as pre-exposure time, temperature rise rate, and isothermal warm-up time. However, the determining factor that has the greatest impact on the water resistance of concrete during heat treatment is the duration of the preliminary exposure and the rate of temperature rise, on which the values of internal stresses in concrete depend on heating.

The duration of the pre-aging is mainly dependent on the properties of the concrete mix. Yu.M.Bazhenov and other scientists [1-5] recommends to carry out preliminary exposure for mobile mixtures for 3-6 hours, for hard mixtures - at least 2-3 hours and for particularly hard mixtures - 1-2 hours. Pre-curing of concrete mixtures with a complex additive, including C-3 superplasticizer (without reducing V/C), may be somewhat larger compared to concrete mixes without additives, i.e. with reduced $\mathrm{W} / \mathrm{C}$, does not change. The results of V.A.Babaev and his command study [6-8] of concrete mixtures with mobility of $\mathrm{OK}=8$ $\mathrm{cm}$ showed that preliminary exposure when using the $\mathrm{C}-3$ additive and preparing the equal mixture with the control composition of the concrete mix is about 3 hours. However, prolonged preliminary incubation is irrational, since affects the turnover of steel forms.

The study of the physicomechanical properties of concrete depending on the heat treatment regimes is very important, especially with the complex additive C-3 + CE 119215 , which is being studied for the first time.

\section{Methods}

In studies, the heat and moisture treatment of concrete was carried out in a laboratory steam chamber with automatic control of the regime. To determine the strength of concrete, cubic samples were made with dimensions of $100 \times 100 \times 100 \mathrm{~mm}$, which were tested at the period of 1,3,7,28 days. After HMT compared with similar characteristics of normal hardening samples.

The kinetics of increasing the strength of concrete with improved water resistance were studied on two compositions: without additives, with $\mathrm{W} / \mathrm{C}=0.58$ and mobility $\mathrm{OK}=3.0$ $\mathrm{cm}$; with the complex additive C-3 + KE119-215 (0.5 + 0.1\% by weight of cement $)$ and the mobility of the concrete mix $2-3 \mathrm{~cm}$ and the volume of entrained air in the amount of $3 \%$. 
In both compositions, the cement consumption was the same and amounted to $350 \mathrm{~kg} / \mathrm{m} 3$, and the sand content in the aggregate mixture $\left(r=\frac{p}{p+s h}\right)$ was also the same, i.e. $r=0,38$

For comparison, the steamed samples with samples of normal hardening were made samples of two compositions without additives and with a complex additive (C-3 + KE119215), which were tested at the duration of $1,3,7$ and 28 days. normal curing.

The preliminary incubation was varied in the range of 1-2 hours. The temperature rise rate was 20 and $27{ }^{\circ} \mathrm{C} /$ hour. The duration of isothermal heating was 6 and 8 hours. Isothermal heating was carried out at 60 and $80{ }^{\circ} \mathrm{C}$. The duration of cooling in all cases was 3 hours.

The increase in the structural strength of concrete in natural conditions and during heat and moisture treatment is divided into two periods. In the first of them, within about $2 \ldots 4$ hours from the moment of molding, the structural strength increases slowly. The second period is characterized by a sharp increase in the growth rate of structural strength, which can be increased even more due to the heat of the humidity treatment. Therefore, in order to improve the quality of concrete, it is recommended to start the heat moisture treatment in the second period. With this in mind, HMT in most cases is carried out after preliminary aging of newly formed concrete. Preliminary keeping of the products before steaming contributes to the formation of the initial structure of the concrete in the absence of temperature deformations and moisture migration, which positively affects the strength and durability of the finished products.

The optimal time of preliminary aging varies from 2 to 10 hours and corresponds to the beginning of the setting of concrete, at which time it acquires a strength of about $0.3 \ldots 0.5$ $\mathrm{MPa}$. After that, the concrete is in a closed or open form, and sometimes after a long time sufficient for pre-curing, with the airborne equipment removed on a pallet, loaded into the installation, where steam is supplied. Steam, as a more heated body, gives off the heat of vaporization to less heated bodies - the material and the installation, heats them, and itself in the form of condensate is removed from the installation. Due to heating, the rate of cement hydration reactions increases dramatically and the formation of concrete accelerates.

Thus, during the preliminary exposure period, the structure of concrete is formed in relatively calm conditions (in the absence of intensive migration of moisture, temperature deformations of the materials constituting concrete, etc.). This structure becomes able to perceive thermal effects when the temperature rises. According to the results of many studies, the optimal time for preliminary curing of concrete should be considered as the period for which concrete acquires a strength of about $0.3 \ldots 0.5 \mathrm{MPa}$.

\section{Discussion}

The results of studies of the kinetics of increase in concrete strength depending on the heat treatment regimes are shown in Fig. 1, that, despite the slight difference in the duration of the preliminary curing ( 1 and 2 hours), there is still a certain difference between them. 
Strength, \%

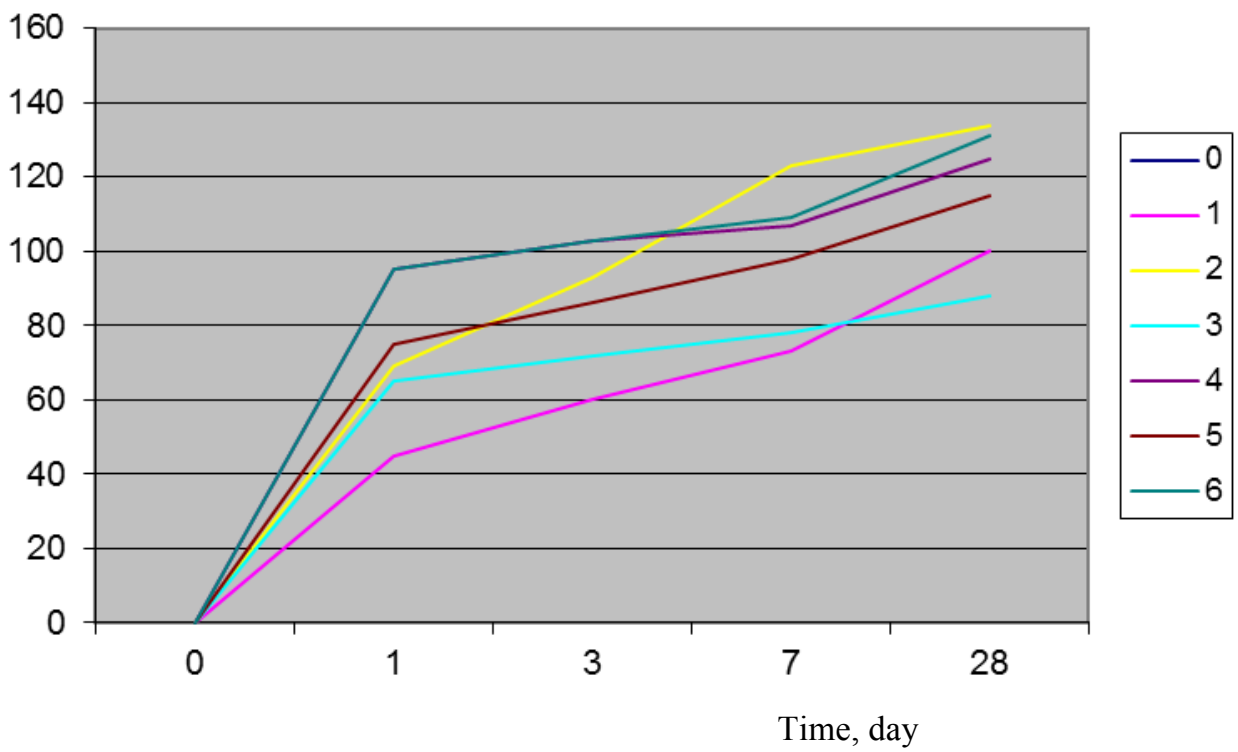

Fig. 1. The kinetics of increasing the strength of concrete, depending on the duration of preliminary aging:

1,3 and 5 samples without additives;

2,4 and 6 - samples with complex additive C - $3+\mathrm{K} Э$ 119- 215 ;

1,2 - normal hardening samples; 3,4 - samples subjected to HMT mode $1+3+6+3 \mathrm{~h} \quad \mathrm{t}=80^{\circ} \mathrm{C} ; 5,6-$ by mode $2+3+6+3 \mathrm{ht}=80^{\circ} \mathrm{C}$.

So, if the concrete without additives with preliminary exposure for 1 hour has after HMT at the period of 28 days. $88 \%$ brand strength, then at 2 hours of preliminary exposure, it reaches $115 \%$. For concretes with complex additive C-3 + KE119-215, the kinetics of increase in strength is the same as for concretes without additives. It should be noted that normal concrete hardening with complex additive C-3 + 119-215 increases strength in the first 7 days compared to concrete without additives.

The reason for this may be a low water-cement ratio and the presence in the concrete of the silicone polymer, which increases the degree of cement hydration, as will be discussed below.

Interestingly, the strength of normal curing concretes with the complex additive C-3 + KE119-215 is 1 day old- $69 \%$, and 3 days old. - $94 \%$, at the period of 7 days. $-123 \%$ of the brand.

The second stage - temperature rise is characterized by speed indicators, which can be from 10 to $30^{\circ} \mathrm{C} / \mathrm{h}$. The earlier concrete gains the minimum structural strength able to withstand the pressure of steam and gaseous products, the greater the rate of temperature rise may be. Consequently, the duration of this period is closely related to the previous one. In the presence of factors contributing to the reduction of the exposure time, the rate of temperature rise can be $30^{\circ} \mathrm{C} / \mathrm{h}$. The maximum temperature depends on many factors.

Heating of products in the first period of heat-moisture treatment occurs through the thermal conductivity of the material, due to convective heat transfer from the vapor-air medium and vapor condensation. The water formed during the condensation of steam is precipitated in the form of droplets on the surface of the product. Since during the rise in temperature, as well as at the beginning of the isothermal period, the product has a lower temperature than the medium, the vapor pressure in the chamber exceeds the vapor pressure in the product; the product absorbs a certain amount of water formed on its surface. An 
increase in the moisture content of the upper layers of still unconsolidated concrete causes the water to move to the center, and sometimes a slight increase in linear dimensions, especially in the upper layers. At the same time, concrete phenomena are observed in the concrete, leading to a decrease in volume and contributing to the migration of water into the concrete.

The effect of temperature at the stage of temperature rise is especially great (since the structure of concrete has not yet formed, and its strength is low) and manifests itself in microdamages of the integrity of the structure of cement stone and at the border of its contact with aggregates. During this period, especially during rapid heating, considerable stresses may arise in the product, as a result of which cracks are formed, and contact between the cement stone and the aggregate is disturbed.

During heat treatment, concrete as a result of thermal expansion increases in volume. As is known, concrete is a composite consisting of dissimilar materials: cement, water, coarse and fine aggregates. In addition, each material that is part of concrete, has its own coefficient of thermal expansion. Of all the components of the composite, water has the largest thermal expansion coefficient, which expands hundreds of times more than a solid skeleton.

Heating of products in the first period of heat-moisture treatment occurs through the thermal conductivity of the material, due to convective heat transfer from the vapor-air medium and vapor condensation. The water formed during the condensation of steam is precipitated in the form of droplets on the surface of the product. Since during the rise in temperature, as well as at the beginning of the isothermal period, the product has a lower temperature than the medium, the vapor pressure in the chamber exceeds the vapor pressure in the product; the product absorbs a certain amount of water formed on its surface. An increase in the moisture content of the upper layers of still unconsolidated concrete causes the water to move to the center, and sometimes a slight increase in linear dimensions, especially in the upper layers. At the same time, concrete phenomena are observed in the concrete, leading to a decrease in volume and contributing to the migration of water into the concrete.

The effect of temperature at the stage of temperature rise is especially great (since the structure of concrete has not yet formed, and its strength is low) and manifests itself in microdamages of the integrity of the structure of cement stone and at the border of its contact with aggregates. During this period, especially during rapid heating, considerable stresses may arise in the product, as a result of which cracks are formed, and contact between the cement stone and the aggregate is disturbed.

During heat treatment, concrete as a result of thermal expansion increases in volume. As is known, concrete is a composite consisting of dissimilar materials: cement, water, coarse and fine aggregates. In addition, each material that is part of concrete, has its own coefficient of thermal expansion. Of all the components of the composite, water has the largest thermal expansion coefficient, which expands hundreds of times more than a solid frame.

If concrete were a mechanical mixture of unrelated materials, the expansion value when heated would be equal to the sum of the products of the volume of each material by its coefficient of thermal expansion, and when cooled, the volume of the mixture would return to its original value. However, after HMT, concretes are prone to residual expansion. This is explained by the fact that during heat treatment in the material there are complex physicochemical hardening processes. With rapid warming up of freshly molded concrete, the physical process of thermal expansion is ahead of the chemical process of hardening. With a slow rise in temperature, more favorable conditions are formed for the formation of the structure: concrete acquires a certain strength before it reaches a significant thermal expansion. 
Migration of moisture in the hardening material contribute to the formation of interconnected porosity and the appearance of internal excessive pressures. Migrating in the concrete between the deep and surface layers, liquid and air connect pores and voids between each other, increase the effective radius of capillaries, destroy not yet strong bonds at the points of contact of spliced crystalline neoplasms, and create porosity (perpendicular to the surface of products). It is generally known that heat and moisture treatment compared with normal moisture curing reduces the density of concrete, and, consequently, increases its porosity.

Studies of the effect of the temperature rise rate on the kinetics of increasing the strength of concrete (Fig. 2) showed that despite the slight difference in the rate of temperature rise (only $7^{\circ} \mathrm{C}$ ), there is a certain difference in strength characteristics..

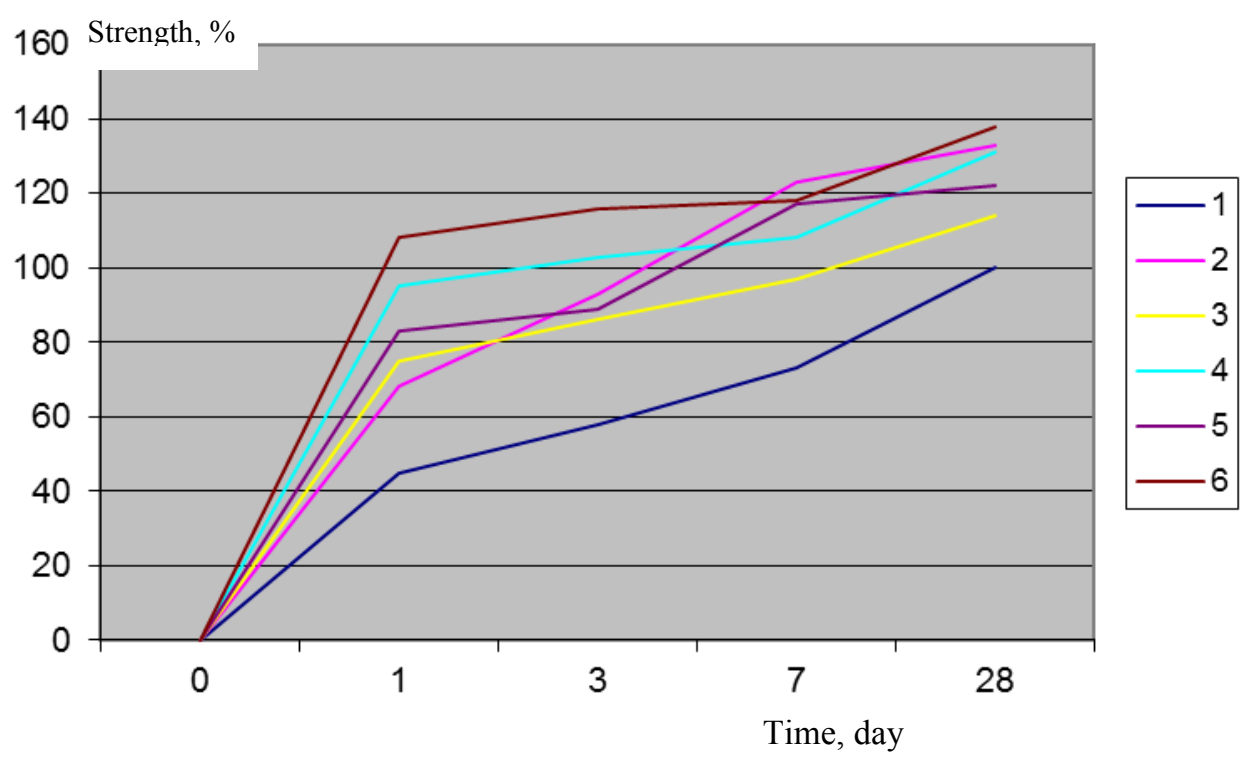

Fig. 2. Kinetics of increasing the strength of concrete, depending on the rate of temperature rise: 1,3 and 5 -samples without additives;

2,4 and 6 - samples with complex additive $\mathrm{C}-3+\mathrm{K} Э 119-215$; 1 and 2 - normal curing samples;

3 and 4 - samples with a temperature rise rate of $27^{\circ} \mathrm{C} /$ hour; 5 and 6 - same $20 \mathrm{C} /$ hour.

So, if with complex additive C-3 + KE119-215 at a rate of temperature rise of $20^{\circ} \mathrm{C} /$ $\mathrm{h}$, it gains strength at the period of 28 days. equal to $138 \%$ of the brand, at a heating rate of $27^{\circ} \mathrm{C} / \mathrm{h}$ it is $7 \%$ lower, i.e. equal to $131 \%$. It should be noted that the strength of concrete at a temperature rise rate of $20^{\circ} \mathrm{C}$ in the first 7 days. increases dramatically in comparison with concretes subjected to heating at a rate of $27^{\circ} \mathrm{C}$.

Isothermal heating of the concrete during its heat-humid treatment, has a nonimmaterial value. Because the highest rate of formation of the structure of concrete is observed in the second period of heat and moisture treatment, during aging at a constant temperature. The differences in temperature and moisture content over the cross section of the material in this period begin to decrease and gradually equalize, which significantly improves the conditions of structure formation. In addition, at this time there is a further hydration of cement. Moisture from the gel formed on the surface is sucked off by the inner layers of the cement grain. Due to the decrease in the moisture content of the gel, the crystallization of the new growth begins, which causes an increase in the processes of structure formation and hardening of the entire system. 
Of particular note is the negative role of air in concrete during the formation of its structure during heat treatment (the content of air involved in the process of preparation and molding is usually $2 \ldots 4 \%$. Air due to expansion puts pressure on the pore walls, trying to move them apart and to occupy a larger volume, but this is possible only when there is no preliminary curing, the concrete has not yet acquired structural strength and its heat treatment takes place without fixing the original size of a rigid metal a common form, and the pressure formed in the pore is enough to push the surrounding layers apart.

The movement of air trapped in concrete under the action of heating is carried out mainly upwards, towards the open surface of the product and the smallest in cross section, which leads to deformation and delamination of the upper layers. However, this process is observed mainly in the HMT of freshly molded products without forms according to the modes with a rapid rise in temperature. When steaming a product in an open form, the air tends to go outside and deforms only the open surface.

The results of the study of the effect of the duration of isothermal heating for 6 and 8 hours showed (Fig. 3) that the kinetics of the increase in strength of concrete with the C-3+ KE119-215 complex additive compared to concrete without additives is practically the same (isothermal temperature of $60^{\circ} \mathrm{C}$ ) . For example, concrete with complex additive C-3 + KE119-215 with an isothermal heating time of 6 hours (Fig. 3) 1 day after HMT gains $77 \%$ of brand strength, the same concrete with an 8 -hour isothermal temperature gains $80 \%$ branded strength.

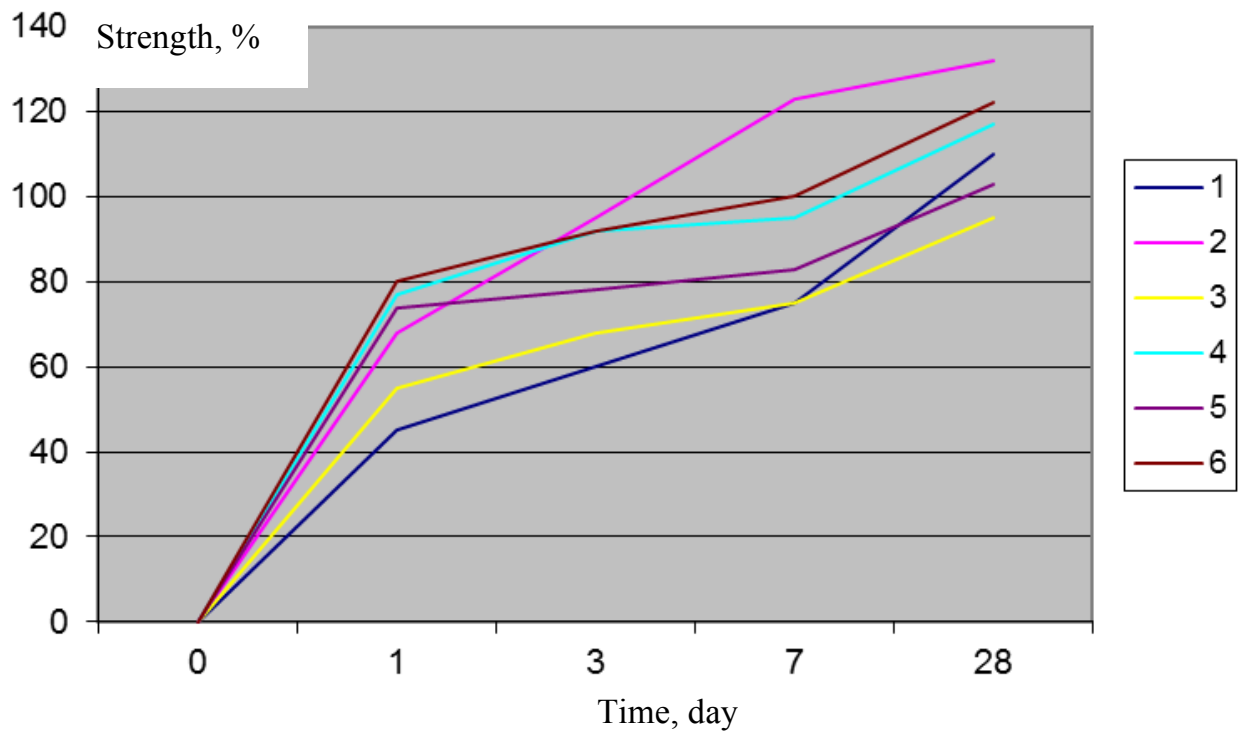

Fig. 3. The kinetics of increasing the strength of concrete, depending on the duration of isothermal heating:

1,3 and 5 samples without additives;

2,4 and 6 - samples with complex additive $C-3+K \ni 119-215$;

1 and 2 - normal curing samples;

3 and 4 samples of isothermal heating for $6 \mathrm{~h}$ :

5 and 6 - the same, $8 \mathrm{~h}$.

The strength of concrete without additives, depending on the duration of heating, is noticeably different, and at 6-hour duration it is 35\%, at 8 -hour- $72 \%$.

This gives grounds to recommend the duration of isothermal heating for 6-hours for concretes with this complex additive. 
The results of the study of the kinetics of increase of concrete strength depending on the temperature of isothermal heating are shown in Fig.4.

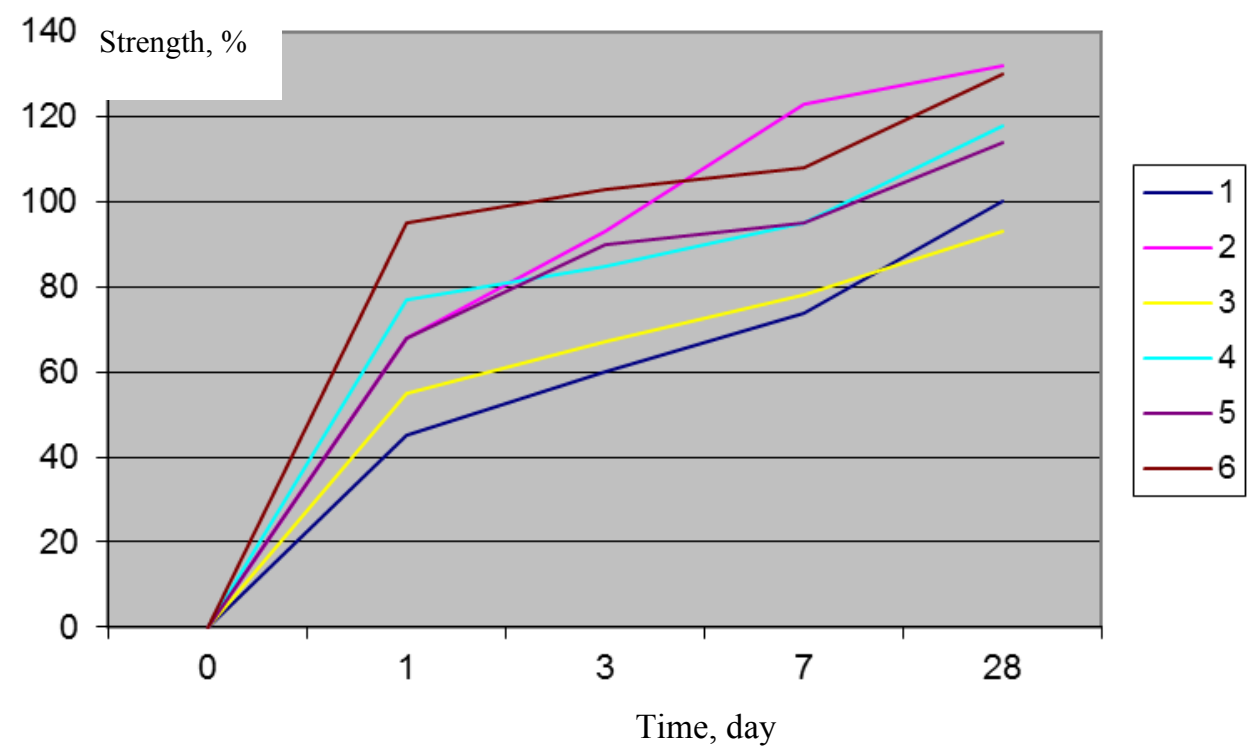

Fig. 4. The kinetics of increasing the strength of concrete depending on the temperature of isothermal heating;

1,3 and 5 samples without additives;

2,4 and 6 - samples with complex additive C-3 + KЭ119- 215;

1 and 2 - normal curing samples;

3 and 4 samples subjected to $\mathrm{HMT}$ isothermal heatingt $=60^{\circ} \mathrm{C} ; 5$ and 6 - the same at $80^{\circ} \mathrm{C}$.

As can be seen from the results of research, the kinetics of increasing the strength of concrete with the complex additive C-3 + KE119-215 and without additives increases with increasing temperature of isothermal heating from $60^{\circ} \mathrm{C}$ to $80^{\circ} \mathrm{C}$. It should be noted that the strength of concrete with a complex additive C-3 + KE119-215 after 1 day. after HMT, with isothermal heating, $80^{\circ} \mathrm{C}$ gains $94 \%$ of the brand strength, and at $60^{\circ} \mathrm{C}, 77 \%$.

It should be noted that the process of cooling after heat-moisture treatment also has an important role. When the temperature in a thermal installation decreases, concrete has a high temperature, and the internal vapor pressure in the product exceeds the ambient vapor pressure $[9,10]$. Due to the existing temperature difference, intensive evaporation of moisture from concrete occurs. The amount of water lost by concrete during heat treatment varies and mainly depends on the composition and density of the concrete, as well as on the mode of heat treatment. According to various researchers, this value can be from 20 to $40 \%$ of the mixing water.

As the product cools and evaporates from the surface, moisture migrates from the central parts of the product. Moisture, moving away from the product in the form of steam, forms channels that run in all directions from the central sections of the product to the periphery and connect the voids and between them. As a result, cement stone has more pores, and after heat treatment is characterized by directional porosity, which increases the permeability of products and reduces their frost resistance. 


\section{Conclusion}

Thus, heat treatment of concrete with increased water resistance (with the complex additive $\mathrm{C}-3+\mathrm{KE} 119-215)$ at a temperature of $60^{\circ} \mathrm{C}$ increases water resistance, frost resistance, and at the same time saves heat and power resources.

Heat treatment of concrete at a temperature of $80^{\circ} \mathrm{C}$ increases the strength of concrete and shortens the overall cycle.

An increase in pre-aging from the 1 st hour to 2 hours increases the strength of the concrete by $15 \%$.

With the complex additive C-3 + KE119-215 at a rate of temperature rise of $20^{\circ} \mathrm{C} / \mathrm{h}$, strength increases at the period of 28 days. equal to $138 \%$ of the brand, at a heating rate of $27^{\circ} \mathrm{C} / \mathrm{h}$ it is $7 \%$ lower, i.e. equal to $131 \%$.

The strength of concrete without additives, depending on the duration of heating, is noticeably different, and at 6-hour duration it is $35 \%$, at 8 -hour- $72 \%$.

This research was supported by Zenatkor Temirbeton Buyumlari LLC on the basis of the contract No. 1482 dated 05.20 .2015 on the topic "Development of recommendations on the heat treatment of concrete for the manufacture of precast concrete products".

\section{References}

1. Bazhenov Yu.M. Concrete technology. -M .: Higher School, 1978.-455 p.

2. Falikman, V.R. New generation superplasticizers Text. / Falikman V.R., Weiner A.Y., Bashlykov N.F. // Concrete and reinforced concrete. 2000. - No.5.

3. Babaevskaya, T.V. Cement concretes modified with complex additives Text. / T.V.Babaevskaya // Author. diss. for the degree of Ph.D. 05.23.05. Odessa, 2003. pp. 19

4. Usherov-Marshak, A.V. Concrete additives: progress and problems Text. / UsherovMarshak A.V. // Construction Materials. 2006. No.10.

5. Izotov, B.C. Chemical additives for concrete modification Text. / B.C. Izotov, Yu.A. Sokolova // M.: Paleotype. 2006. pp..244.

6. Babayev V.A. Heat and moisture treatment of concrete with the addition of superplasticizers C-3 // Concretes with effective superplasticizers. - M., 1979. - pp. 6985.

7. Fedosov, C.B. Influence of heat and moisture treatment on the performance properties of concrete / C.B. Fedosov, S.M. Bazanov, M.V. Akulova, M.V. Toropova // Izv. universities. Building. 2003. - No.7. - p. 47-50;

8. Kalashnikov, V.I. Effect of heat treatment on the kinetics of high-strength concrete curing / V.I. Kalashnikov, B.C. Demyanova, N.M. Duboshina // Izv. universities. Building. 2000. - №2-3. - pp. 21-25;

9. Lesovika, B.C. Concrete on composite binders / B.C. Lesovika, M.S. Ageeva, N.I. Alfimova // Concrete and reinforced concrete. St. Petersburg .- 2012. - No.2 (7). - p. 99101;

10. Batrakov, V.G. The problem of corrosion of modified concrete / VG Batrakov // Concrete and reinforced concrete. Moscow. - 2006. - No.6. - p. 30-31; 